\title{
ImERSE (Improving Experience through Regular Shadowing Events)
}

\author{
William Calvert, Joanne Minford, Carol Platt, Catriona Chatfield
}

Alder Hey Children's NHS Foundation Trust

\begin{abstract}
Systematic operational quality improvement strategies within the NHS are hard to find, although there are numerous published reports of sporadic departmental models and methods resulting in improvements in clinical care. We describe the experience of devising a tool to provide large data collection of patient care experiences by using medical students to shadow patient journeys. This combines patient and family centred care (PFCC) and quality improvement approaches to create a systematic organisational strategy for improving care.

The ImERSE (improving experience through regular shadowing events) approach could be applied to any area of health care to generate population specific improvement priorities. It can be used to promote patient and family centred care and provide a unique medical education experience.
\end{abstract}

We describe its evolution in its first year of use and suggest that using the ImERSE approach delivers beneficial characteristics to patients and their families, those undergoing a shadowing experience, and provider organisations.

\section{Problem}

In 2008 Lord Darzi concluded that "quality" should be "at the heart of the NHS".[1] The NHS Constitution update of 2013 reinforced this statement by stressing increasing patient involvement throughout its seven core principles. Despite these governing principles, findings within the 2013 Francis Inquiry were of Trusts where the accountability to the patient had been lost, Trusts that "had no culture of listening to patients" and where "patients and relatives felt excluded from effective participation in the patients' care".[2] These findings highlighted the need for a national refocus on putting patients at the centre of decision making and quality improvement.

At Alder Hey Children's NHS Foundation Trust we have a background of patient and family centred care (PFCC) projects that started with a Child Health and Family Forum (CHAFF) and more recently has resembled a collaboration with The King's Fund to use PFCC methodology to capture the experience of patients presenting to the accident and emergency department with abdominal pain. The result was the development of an acute abdominal pain pathway which guides the patient journey from triage to a newly formed surgical decision unit. When we reviewed the success of this project we realised that there was the potential to apply this more widely. The goal of our project therefore was to promote PFCC across our organisation and to use this approach to improve patient care.

\section{Background}

Family centred care as an over-riding principle has long been practiced within paediatrics and is considered by some to be a gold standard of care.[3] In adult practices patient-centred care has a long background of evidence within oncology, palliative care and intensive care settings. $[4,5,6]$ Patient and family-centred care
(PFCC) is the natural progression from these two practices and an international institute (Institute for Patient and Family Centred Care) was established in 1992 and acts as an information centre for those wishing to evolve a practice of PFCC. PFCC is a healthcare concept which promotes compassion, respect, and dignity in providing a service that sees the patients and their families as partners and collaborators. "It is care that revolves around the needs and desires of patients and families rather than around the organizations and systems in which it is provided".[7] Within the USA the University of Pittsburgh Medical Centre (UPMC) in Pennsylvania is at the forefront of PFCC practices and has gone to lengths to define the data collection methodologies that embody the principles of PFCC. $[8,9]$ We wanted to ensure that within our tertiary paediatric hospital we practiced and promoted the concept of PFCC and were able to reflect this within our operational and quality improvement strategies.

A systematic literature review of NHS Library databases AMED, BNI, CINAHL, EMBASE, Health Business Elite, HMIC, Medline for the search criteria ("patient and family centred care" and quality improvement) and (PFCC and quality improvement) revealed four returns - appraisal of these reveals one audit to UK practicing standards in a hospice in Hong Kong, [11] a discussion of the role of the family meeting in oncology services in Australia,[12] a dissertation surrounding nursing involvement in PFCC [13] and a paper from Michigan describing the importance of implementing a PFCC steering group.[14]

Expanding the search to (patient and family centred care AND quality improvement) of the same databases returned 48 papers. When duplicates were removed there were 30 for consideration, but initial appraisal and exclusion due to non-UK location, RCT, or single clinical scenario and intervention reduced this to 11 studies for consideration. Oxford University based Locock draws attention to the cost implication of experience based co-design where a 
qualitative video interview and analysis is used to promote PCC.[15] Two other significant studies within the NHS are Ewart et al who report of an audit to promote PFCC on an adult cardiac ward [16] and Shaw et al from Liverpool Heart and Chest Hospital who describe patient shadowing by staff members as a valuable tool for developing insight into the patient experience, but do not make any reference to the principle as a quality improvement tool.[17] However they are considered, these papers are of isolated clinical applications of PFCC.

\section{Baseline measurement}

This project was not set up in response to an identified problem at Alder Hey. It was devised to see if we could promote patient and family centred care whilst improving care pathways through cycles of continuous improvement. The expected findings were evidence which confirmed the service inconsistencies that healthcare professionals know exist. We also hoped to identify improvements that were of significance to patients but that could not be easily identified by healthcare professionals working within the system. We did not know if PFCC methodology could be used to promote quality improvement on a large scale but we did know that it could be used for small scale change.[10, 8$]$

\section{Design}

In the absence of any NHS based published work of systematic organisational change through PFCC practices we decided to design an approach ourselves. Review of PFCC methodology and practices reveals three methods of data collection in relation to patient experience. These are patient and family shadowing, patient and family story-telling, and surveys.[8,9] The idea from these is to produce care experience flow maps as a means to identify areas of care provision for improvement cycles.

The task which we set ourselves, devising a tool for systematic organisational quality improvement, requires the collection of a large volume of data. In order to do this we had to look for the most reproducible method of collecting data through a PFCC method. However, we were also wary of replacing the primary goal of producing better care with a quantitative data collection exercise. We realised that the most accessible method for large volume qualitative data collection was patient shadowing as described first by DiGioia in 2007.[8] The resources for designing a shadowing system are well described by both DiGioia and the King's Fund.[18, 19] This means that the extra burden on staff of conducting shadowing events was something we felt was unlikely to be received positively. We therefore looked for another population within Alder Hey capable of carrying out the shadowing events - the obvious choice was medical students, tomorrow's doctors.

Liaising with the undergraduate coordinators, we discovered that all third year medical students undertook a shadowing event of a daycase surgical pathway, following a patient and family from their admission to the daycase ward, through surgery and on to discharge. However, there was no return for their time against identified learning outcomes or data generation for the Trust. At that time the number of participants over the course of a year was between 250 and 300 students. We gained support from the executive board and the go ahead from the University of Liverpool school of medicine undergraduate department to try to incorporate a quality improvement component into their daycase experience. This led to the development of ImERSE (improving experience through regular shadowing events).

Challenges recognised in the design process included implementing the practicalities of the programme, engaging with the medical students and deciding how to analyse the collected data. We did not know if analysing qualitative data on such a large scale was possible and if it was whether any meaningful metrics for improvement could be ascertained form it. Clinical quality improvement in the format of audit and morbidity and mortality meetings is done well within the NHS, and there have been previous theories that the lack of involvement of practicing clinicians in organisational quality improvement is what has led to their limited success. We therefore ensured that ImERSE was a clinician designed and implemented program, with the support of the service improvement lead. We felt that the buy-in from the medical students from the outset would depend on the visible presence and support of clinicians.

\section{Strategy}

PDSA cycle 1: Our first ImERSE cohort consisted of 40 students. We produced a data capture sheet for the students and they were encouraged to record directly observed real time feedback on the care experience touchpoints they witnessed. They were encouraged to make their observations objective from their viewpoint as an observer, and also subjective from the patients and their families viewpoint following the establishment of an open relationship of two way communication. The students captured their observations in a pre-designed table and also produced a reflective writing report.

These initial observations underwent a brief analysis looking for theme recurrences or negative inconsistencies, at this stage it was done to confirm that the ImERSE approach was a suitable means of data collection for quality improvement. We also held a feedback session with the students, following a majority affirmation of its usefulness and based on some of their comments we adapted the data collection sheets to be clearer and better structured. Other initial feedback comments were surrounding more of a tutorial basis to shadowing and also ensuring that the wards were aware of the module.

PDSA cycle 2: Confident with the overall format, but appreciating that a finished article was a number of cycles away we continued with ImERSE through the Lent and Summer terms until the summer break of 2014. Each cohort of medical students (cohort size 35 to 40) was given an introductory lecture; within this we include a guide to shadowing. We also identified patient shadowing advocates within the daycase ward to facilitate the students, and also developed a FAQ sheet for the students to give to the families explaining the ImERSE approach and the role of the students as shadows. All of these changes were based on feedback from the 
medical students gained at formal debriefing sessions.

Through these cycles we also turned out attentions to more robust thematic analysis of the observation and reports. This was done through scrutiny analysis, by being able to develop a system that was quick and robust enough not to miss anything of importance we are able to feedback to each student cohort their observations. We also feedback to the surgical daycase ward at regular intervals, and also run longitudinal analyses to ensure that quality improvement changes continue to be evidenced in practice. Some of our initial findings were used to clarify communication around the administration of pre-medication and also resulted in the supplying of a television for the parent waiting area.

PDSA cycle 3: By the end of the summer term, we had witnessed a few quality improvement changes on the daycase ward, brought about by feedback of observations extrapolated from ImERSE. We had also begun to realise a wider reaching medical education approach to the tool. In the months of August and September 2014 we identified five key areas of learning mainly surrounding areas of the "hidden curriculum" and quality improvement and incorporated details of these expected learning outcomes into out introductory talks.

These educational benefits, which were in fact originally identified as a secondary aim behind improving care and generating a person centred approach to quality improvement, are now some of the main benefits of the programme. This has led us to develop ImERSE as a formal module alongside the undergraduate department of the University of Liverpool school of medicine at Alder Hey. This means that more senior students in their fourth or fifth years who are returning to Alder Hey can undertake shadowing experiences in different clinical situations (ie the emergency department) and through a vertically integrated curriculum can continue learning about quality improvement and PFCC in more depth.

PDSA cycle 4: Following the identification of clear learning outcomes we were able to devise more intricate environments where patient shadowing could take place. This has led to an extension of ImERSE into the accident and emergency department and we are in the design stage of an outpatient model. Now in its second academic year, the ImERSE approach appears to be sustainable due to its strength of medical education and quality improvement making it an attractive module for medical students at Alder Hey.

The rapid through-put of students (a new cohort every five weeks) allows us to continue to improve the experience of the ImERSE programme quickly in response to their feedback.

We have also recently been successful in adapting the proforma to enable us to perform specific data capture tasks such as auditing the adherence to the WHO Surgical Safety checklist and assessing how time of clinical review impacts start time for daycase theatres.

\section{Results}

The key to the success of this project was developing a rigorous method of analysing the data. The data collected was qualitative with the main aims being to understand the perspectives of patients and their families, explore the meaning they gave to phenomena that occurred through their care experience and extract themes for quality improvement.

We established that the short textual data recorded verbatim would require thematic analysis.

Expanding on previously reported findings from shadowing and based on the volume of data we are able to collect, we continue to utilise a scrutiny technique of analysis looking for recurring regularities (positive consistencies), recurring irregularities (negative inconsistencies), we also use normal and abnormal word co-occurrence as a method of identifying singular irregularities. The identification of negative inconsistencies from shadowing has previously been described by Dr DiGioia.[8] The validity of the analysis is based on the judgement of the analyser who has significant past experience in thematic analysis techniques and access to the clinical members of the team when needed for validity purposes.

Following each cohort of students, the real time documentation and reports were thematically analysed for themes that could inform service improvement. We also identified themes which represented positive feedback for staff and services. This positive feedback helps to enforce a message of striving for excellence and not simply focusing on aspects of service that could be improved. The nature and timing of the data collected through ImERSE means that we produce reports of findings on a monthly basis that can be fed back into care at a clinical level. It also allows us to report greater risk findings at the same frequency at executive board level. Serial monthly analysis enables us to map the effect of changes made in response to our student, patient and family feedback by trend analysis for its recurrence in future cohorts of data collection. That is, if the themes do not recur within future cohorts we can extrapolate that the aspect of care reported has been successfully improved.

So far we have implemented small scale changes within the parents waiting area, and addressed communication surrounding the dispensing of pre-medications. This change led from the abnormal word co-occurrence of "random drug" written by a student, further analysis of this observation and others in that cohort (this was the second student cohort) identified the communication surrounding pre-medication administration as an area of concern. This was discussed with the daycase unit and subsequent cohorts of observations provided a method of continuous observation of the improvement of pre-med dispensing.

\section{Lessons and limitations}

The results from the initial student cohorts undergoing ImERSE proved to be extremely exciting. We have the identified two major benefits from the project. Using a quality improvement method with PFCC methodology as its primary source of data collection we are able to move towards creating patient centred and also population 
specific care experience pathways where the negative inconsistencies of care are identified by patients and acted upon systematically within the organisation.

The second benefit is in relation to medical student education. We know that authentic early years' experience [20] and increased shadowing events decrease starter anxiety [21] in the key area of preparing for practice and ImERSE fulfils both of these requirements. It can be used to support interprofessional education and deliver patient safety and quality improvement at an undergraduate level.

We haven't struggled with clinical buy-in in our experience, the patient and family centred care (PFCC) leads for Alder Hey are an emergency department consultant and a paediatric surgical consultant, and ImERSE has received Trust board support and the support of the associate medical director (director of education). Our PFCC council who were established preceding the advent of ImERSE included the COO, PFCC clinical leads, service improvement lead and head of transformation.

We have found that the majority response from the students is positive. Over $90 \%$ rate it a beneficial experience with regards their future careers. The remaining percentage who don't are those we are actively trying to engage with and involve in ongoing improvement. Despite the researched and developed academic proposal behind ImERSE we are aware that a limitation of it's is the lack of validation of the educational benefits. This is something that we are currently trying to explore.

\section{Conclusion}

ImERSE represents a powerful tool for systematic organisational quality improvement, being robust enough to identify positives for staff morale boosting and risk behaviour severe enough to end in never events. It also provides a unique and valuable insight for students into patient and family centred care and the "hidden curriculum". By increasing awareness and authentic exposure to care experiences we can decrease "starter anxiety" as students progress through medical school and also increase awareness of the importance of the experiences they will have to enable them to think towards establishing their own professional identities.

Further development of ImERSE includes external validation of the placement as a method of empathy acquisition in medical students and external validation of patient and family centered care as a method of driving organisational quality improvement.

\section{References}

1. Darzi A. High Quality Care for All: NHS Next Stage Review (Final Report). Department of Health London. 2008; Gateway reference 10106.

2. Francis, R. Report of the Mid Staffordshire NHS Foundation Trust Public Inquiry Executive summary. 2013. London: Crown Copyright.

3. Jolley J, Shields L. The Evolution of Family Centered Care.
J Paediatr Nurs 2009;24(2):164-70.

4. Zucca A, Sanson-Fisher RW, Waller A, Carey M. Patientcentred care: making cancer treatment centres accountable. Support Care Cancer 2014;22(7):1989-97.

5. Mackenzie LJ, Carey ML, Sanson-Fisher RW, D'Este CA. Perceptions of patient-centred cancer care during radiotherapy treatment: A cross sectional survey. AsiaPacific J Clin Oncol 2012;(8):263-4.

6. Hynes P, Conlon P, O'Neill J, Lapinsky S. Partners in critical care. Dynamics (Pembroke, Ont.) 2008;19(1):12-7.

7. www.pfcc.prg/what-is-pfcc/

8. DiGioia AM, Greenhouse PK, Levison TJ. Patient and Family-centered Collaborative Care - An Orthopaedic Model. Clin Orthop Relat Res 2007;(463):13-9.

9. DiGioia AM, Greenhouse PK. Patient and family shadowing: creating urgency for change. J Nurs Admin 2011;41(1):23-8.

10. Hast A, DiGioia A, Thompson D, Wolf G. Utilizing complexitiy science to drive practice change through Patient- and Family-Centre Care. J Nurs Admin 2013;43(1):44-9

11. Shaw J, Pemberton S, Pratt C, Salter L. Shadowing: a central component of patient and family-centred care. Nurs Manag (Harrow) 2014;21(3):20-3.

12. Ming MH. Audit of multi-disciplinary care in a Hong Kong hospice. Palliative Care Today 1998;7(1):12-3.

13. Savva J, McLeod V, Jones M, Mair K, Harvey A, Megee F, et al. The family meeting cocktail: A recipe for interdisciplinary action. Program in Detail. Asia-Pacific J Clin Oncol 2009;5(s2):A104-38.

14. Peterson KS. Measuring hospital climate for the delivery of patient- and family-centered care. 2009. Brandeis University, The Heller School for Social Policy and Management.

15. Commins J. Staffing for Better Outcomes. Health Leaders Magazine 2010;13(5):55-6.

16. Locock L, Robert G, Boaz A, Vougioukalou S, Shuldham C, Fielden $\mathrm{J}$, et al. Testing accelerated experience-based codesign: a qualitative study of using a national archive of patient experience narrative interviews to promote rapid patient-centred service improvement. Health Serv Del Res 2014;2(4).

17. Ewart L, Moore J, Gibbs C, Crozier K. Patient- and familycentred care on an acute adult cardiac ward. Brit J Nurs 2014;23(4):213-8.

18. www.pfcc.org/get-started

19. http://www.kingsfund.org.uk/sites/files/kf/field/field_pdf/pfcctoolkit.pdf

20. Yardley S, Teunissen PW, Dornan T. Experiential learning: AMEE Guide No. 63. Medical Teacher 2012;34(2):e102-15.

21. Kavanagh P, Boohan M, Savage M, McClusky D, McKeown $P$. Evaluation of a final year work-shadowing attachment. Ulster Med J 2012;81(2):83-8.

\section{Declaration of interests}

Nothing to declare. 


\section{Acknowledgements}

Graham Lamont, Judith Adams.

\section{Ethical approval}

ImERSE was reviewed by the NRES Committee North West Liverpool Central. Their conclusion was that as it was "A service improvement project here that collects qualitative data for internal quality improvement" so no ethics approval was required. 